\title{
Comparação entre a cifose torácica de idosos sedentários e praticantes de atividade física pelo método flexicurva
}

\author{
Comparison of thoracic kyphosis between sedentary and physically \\ active older adults by the flexicurve method
}

Resumo - A hipercifose torácica é uma das alterações posturais mais comuns, sendo definida como um aumento da curvatura torácica no plano sagital da coluna vertebral. Com o envelhecimento, ocorrem alterações no sistema de controle postural que influenciam a mobilidade funcional e o déficit de equilíbrio em idosos. O objetivo do estudo foi comparar a cifose torácica de idosos praticantes de atividade física com idosos sedentários, determinando, assim, a influência da atividade física na cifose dorsal do idoso. Através de um estudo transversal, avaliaram-se 40 voluntários de ambos os sexos e sadios, que foram divididos em dois grupos, um de praticantes de atividades físicas (G1) e outro de sedentários (G2) com idade a partir de 60 anos e inferior a 69, que foram alocados aleatoriamente para aplicação do método flexicurva. Para a análise foram utilizados estatística descritiva, com média e desvio padrão, e o teste t independente de Student. Verificou-se que os indivíduos praticantes de atividade física foram os que menos sofreram com as alterações da curvatura da cifose torácica, mas não houve diferença significativa entre os grupos, indicando que idosos ativos e sedentários possuem características similares quanto à cifose torácica. Podese concluir que idosos ativos e sedentários apresentam características similares quanto à cifose torácica.

Palavras-chave: Cifose; Idosos; Sedentários; Atividade física; Flexicurva.

1 Universidade Católica de Brasília. Curso de Fisioterapia. Brasília, DF. Brasil.

Recebido em 06/04/09 Revisado 23/11/09 Aprovado 05/01/10
Abstract - Thoracic hyperkyphosis is one of the most common postural changes, which is defined as an increase of thoracic spine curvature in the sagittal plane. The alterations that occur in the postural control system with age influence functional mobility and result in a lack of balance in older adults. The objective of this study was to compare thoracic kyphosis between physically active and sedentary older adults in order to determine the influence of physical activity on dorsal kyphosis in the elderly. In a cross-sectional study, 40 healthy volunteers of both genders, ranging in age from 60 to 69 years, were randomly divided into a physically active group and a sedentary group and evaluated by the flexicurve method. Descriptive statistics (mean and standard deviation) and the Student t-test for independent samples were used for analysis. The results showed that physically active older adults presented fewer changes in the curvature of the thoracic spine than sedentary subjects, but the difference was not significant. In conclusion, active and sedentary older adults present similar characteristics in terms of thoracic kyphosis.

Key words: Kyphosis; Sedentary; Older adults; Physical activity; Flexicurve. 


\section{INTRODUÇÃO}

Segundo o Instituto Brasileiro de Geografia e Estatística (IBGE), com base no Censo 2007, o país tem 11.042 pessoas com 100 anos ou mais. Destes, 7.950 são mulheres. Número que é mais do que o dobro dos homens, que somam 3.472 pessoas. Segundo o IBGE, os avanços médicos e as melhorias nas estruturas sociais têm proporcionado ganhos de sobrevida e, como consequência, geram esse aumento da longevidade da população idosa no Brasil ${ }^{1}$.

Em decorrência do envelhecimento, os tecidos que envolvem as articulações sofrem alterações que diminuem sua elasticidade. Entre os 20 e os 70 anos de idade, dependendo da articulação estudada, podem ocorrer declínios de 20\% a 50\% na amplitude de certos movimentos ${ }^{1-3}$. A diminuição/ redução da flexibilidade, particularmente nas articulações da coluna, do quadril e dos joelhos, está associada a dificuldades na realização das AVD's, podendo ser a principal causa de desconforto e incapacidade no idoso 4,5 . Além disso, desvios posturais importantes passam a influenciar a qualidade de vida desta população, pois sua diminuição, além de reduzir a possibilidade de movimento, aumenta o risco de lesões nas articulações ${ }^{6}$.

A hipercifose é uma das alterações posturais mais comuns, o ângulo da cifose torácica pode variar, normalmente, entre $20^{\circ}$ e $40^{\circ}$ na população em geral ${ }^{7}$, no entanto para idosos pode ser considerados como normais ângulos de até $56^{\circ 8}$. Em relação aos idosos, ângulos de até $56^{\circ}$, para o intervalo de cifose torácica, podem ser considerados normais

Desta forma, não existe consenso em relação aos limites de normalidade para curvatura torácica em idosos. Essa deformidade resulta em problemas mecânicos causados pelos diferentes posicionamentos das vértebras em vista sagital, que pode levar a sequela neurológica tardia. Além disso, pode ocorrer déficit respiratório, por reduzir a capacidade de sustentação da coluna vertebral e também a diminuição da expansibilidade torácica ${ }^{7-10}$.

Com o envelhecimento, ocorrem alterações no sistema de controle postural que influenciam a mobilidade funcional e o déficit de equilíbrio em idosos. As alterações dos sistemas músculo-esquelético, neuromuscular e sensorial são os grandes responsáveis pelos elevados índices de quedas na população idosa ${ }^{10}$. Dentre as consequências das quedas nesta população, citam-se o medo de cair novamente, fraturas, principalmente a de quadril, diminuição da autonomia e independência, aumento dos custos com a saúde e até mesmo a morte ${ }^{11}$.
Algumas alternativas podem ser adotadas no intuito de maximizar a mobilidade funcional e o equilíbrio dos idosos, contribuindo, assim, na redução do risco de quedas, dentre elas a reorganização do ambiente do idoso, revisão do esquema medicamentoso e a prática de atividade física ${ }^{12}$. Atualmente, está comprovado que quanto mais ativa é uma pessoa menos limitações físicas ela tem. Dentre os inúmeros benefícios que a prática de exercícios físicos promove, um dos principais é a proteção da capacidade funcional em todas as idades, principalmente, nos idosos ${ }^{13-15}$. A atividade física regular é um recurso importante para minimizar as alterações negativas do envelhecimento,ou seja, possibilita para o idoso uma vida mais ativa, melhorando o bem-estar funcional do organismo e, também, influencia nas atividades da vida diária. A prática de atividade física também promove a melhora da composição corporal, a diminuição de dores articulares, o aumento da densidade mineral óssea, a melhora da utilização de glicose, a melhora do perfil lipídico, o aumento da capacidade aeróbia, a melhora de força, de flexibilidade, a diminuição da resistência vascular ${ }^{5,16}$.

Este estudo objetiva comparar a cifose torácica de idosos praticantes de atividade física com idosos sedentários, pelo método Flexicurva, pois os métodos radiográficos são caros e expõem os sujeitos à radiação, não sendo o método mais adequado para o acompanhamento periódico do paciente, determinando, assim, a influência da atividade física na cifose dorsal do idoso.

\section{PROCEDIMENTOS METODOLÓGICOS}

Esta pesquisa consistiu em um estudo do tipo transversal, no qual uma específica população de idosos, em determinado momento, teve a relação analisada partindo da causa para o efeito, retratando a relação entre as variáveis ${ }^{17,18}$.

O estudo foi desenvolvido no projeto de extensão da Universidade Aberta à Terceira Idade (UnATI) que se encontra na Universidade Católica de Brasília (UCB). A UnATI dispõe de 835 idosos matriculados em diversas atividades, deste total, as mulheres representam em torno de $85 \%$ desta população. A amostra foi composta por 40 idosos voluntários saudáveis, de ambos os sexos, com idade entre 60 a 69 anos escolhidos de forma aleatória e selecionados por convite pessoal. Estes 40 sujeitos foram divididos em dois grupos compostos por 20 integrantes em cada grupo, sendo um grupo composto por idosos sedentários (GS) e outro grupo 
constituído por idosos praticantes de atividade física (GAF).

O presente estudo foi aprovado pelo Comitê de Ética e Pesquisa da Universidade Católica de Brasília sob registro número $84 \mathrm{CEP} / \mathrm{UCB} / 2008$. Todos os participantes do estudo, ou seus responsáveis, leram e assinaram um termo de consentimento livre esclarecido, concordando em participar da pesquisa. As coletas de dados foram realizadas no Laboratório de Biomecânica da Universidade Católica de Brasília, e os critérios de inclusão adotados foram: a) indivíduos com idade entre 60 a 69 anos; b) estar em boas condições físico-mentais, onde foi realizado o mini-exame de estado mental; c) manifestar livre interesse em participar do estudo. Utilizou-se o método Flexicurva no presente estudo, pois é um método que não expõe o paciente à radiação, não é invasivo, de fácil aplicabilidade, de baixo custo e serve como forma de diagnóstico e indicador de evolução de tratamento em estudos de campo, grandes populações e em idosos.

Foram excluídas do estudo pessoas com idade inferior a 60 anos e com idade superior a 69 anos, pessoas que estivessem impossibilitados de ficar na posição ortostática e aquelas que tivessem realizado algum procedimento cirúrgico na coluna vertebral que interferisse de alguma maneira no padrão da cifose torácica.

A faixa etária escolhida deve-se ao fato de que o questionário International Physical Activity Questionnaire (IPAQ), versão curta, aconselhado pela Organização Mundial de Saúde (OMS) para definir os grupos de idosos sedentários e praticantes de atividade física é recomendado somente até o limite de 69 anos. O instrumento contém perguntas relacionadas à frequência (dias por semana), duração (tempo por dia) e intensidade (leve, moderada e vigorosa) da atividade física nos últimos sete dias. Os indivíduos são classificados em muito ativos, ativos, irregularmente ativos e sedentários, de acordo com o escore obtido no IPAQ ${ }^{19}$.

Os 40 sujeitos selecionados foram avaliados por dois pesquisadores devidamente treinados para todas as etapas do trabalho. Sendo 14 do sexo feminino no grupo de sedentários (GS) e 20 no grupo de praticantes de atividade física (GAF), enquanto para o sexo masculino obteve um total de 6 sujeitos, todos alocados no GS. Os participantes foram recrutados com horário marcado, em média de 20 minutos de intervalo para cada coleta de dados.

Estando dentro dos padrões de inclusão da coleta, os participantes eram orientados a vestir um jaleco com abertura nas costas, cedido pelos pesquisadores responsáveis, mantendo exposto apenas à região dorsal a ser avaliada. Esse processo ocorreu para que a medição da cifose torácica fosse realizada diretamente sobre a pele do sujeito.

Foi solicitado a cada voluntário que permanecesse em posição ortostática, descalço, com os braços ao longo do corpo, com alinhamento de membros inferiores e em sua postura habitual. Os voluntários foram orientados, ainda, a descarregar o peso corporal igualmente sobre ambos os pés. Os pesquisadores posicionaram-se atrás dos voluntários para localizar e marcar com etiqueta adesiva nos processos espinhosos da sétima vértebra cervical (C7) e da décima segunda vértebra torácica (T12). Logo após este procedimento, a régua flexível era, então, moldada sobre o dorso do voluntário (figura 1$)^{11}$.
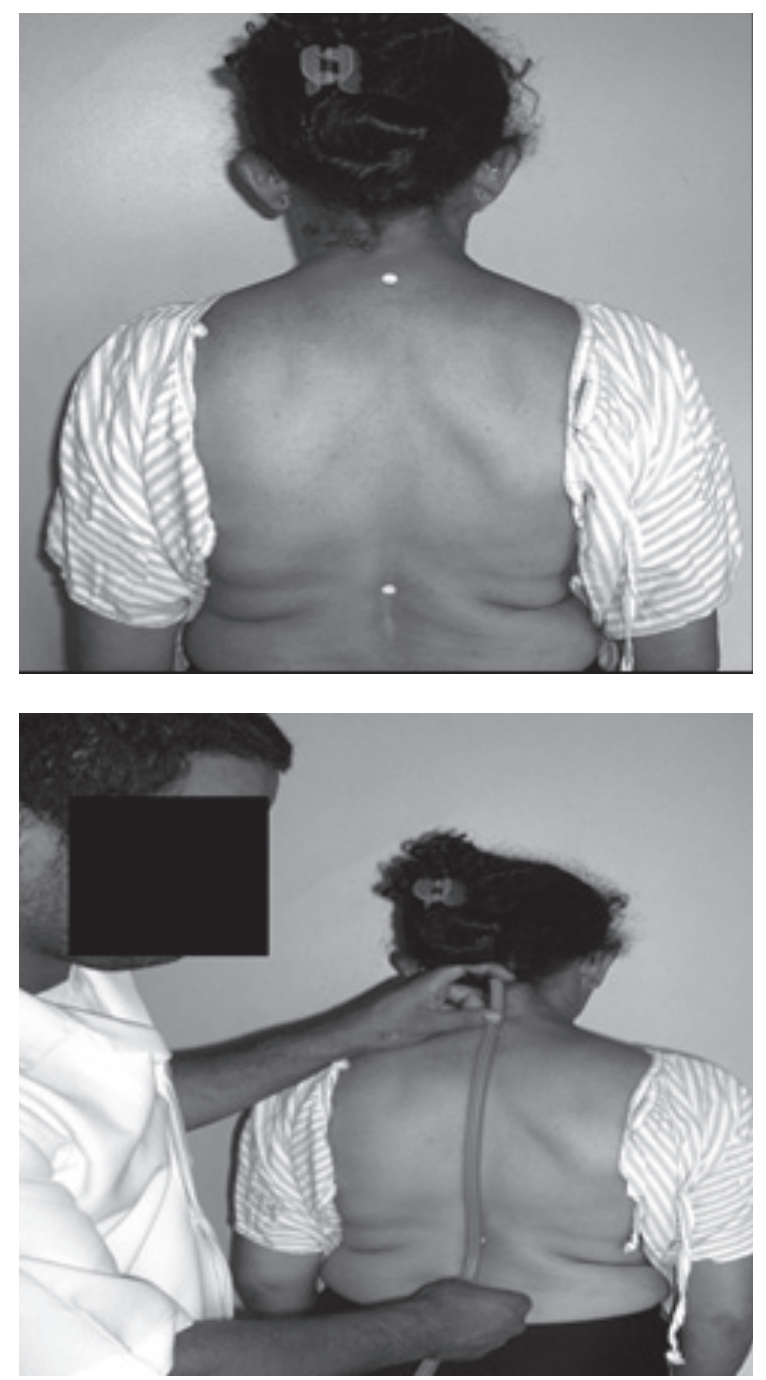

Figura 1. Marcação dos pontos de C 7 e T 12 da voluntária e molde da régua na coluna torácica.

Fonte: autores, 2008

A régua flexível utilizada foi da marca Trident ${ }^{\circledR}$ de $60 \mathrm{~cm}$ de comprimento e o papel milimetrado da marca Canson ${ }^{\circledR} 90 \mathrm{~g} / \mathrm{m} 2$ A2 420 X 594 mm. Logo 
após, foi demarcado na própria régua os pontos referentes aos processos espinhosos de C7 e de T12. O contorno da curvatura cifótica assumido pela régua foi transferido para um papel milimetrado. Este método não calcula diretamente a curvatura cifótica em graus: utiliza centímetros como unidade de medida, que é transferido para um software específico desenvolvido por Teixeira e Carvalho ${ }^{11}$, gerando, então, os valores angulares. $\mathrm{O}$ estudo de Fon et al. ${ }^{8}$ serviu como referência para definição da cifose normal no idoso que é até $56^{\circ}$.

Nos procedimentos estatísticos, foram utilizados a estatística descritiva para caracterização da amostra e o teste $t$ independente para comparar a média da cifose torácica de idosos sedentários com os praticantes de atividade física. Os dados foram plotados nos softwares Excel e SPSS versão 14.0, ambos compatíveis com o Windows. O nível de significância adotado foi igual ou inferior a $5 \%(\mathrm{p} \leq 0,05)$.

\section{RESULTADOS}

Foram, então, analisados os ângulos das cifoses de 40 sujeitos, todos na faixa etária entre 60 e 69 anos, com média de idade de 65,58 anos (desvio-padrão de $\pm 3,13)$.

Houve um maior predomínio do sexo feminino ( $\mathrm{n}=34$ ), sendo 14 no grupo de sedentários (GS) e 20 no grupo de praticantes de atividade física (GAF), enquanto o sexo masculino obteve um total de 6 sujeitos, todos alocados no GS, a média de idade nos homens foi de 64,16 anos e desvio-padrão \pm 3,06 , enquanto a média de idade nas mulheres foi de 65,82 anos e desvio-padrão $\pm 3,12$.

No GS, observou-se uma média de idade de 64,4 anos (desvio-padrão de $\pm 3,12$ ), enquanto no GAF, observou-se uma média de 66,75 anos (desviopadrão $\pm 2,73)$.

A média total das mensurações angulares da cifose torácica dos dois grupos, por meio do método Flexicurva, foi de $47,39^{\circ}$ e desvio- padrão de \pm $10,13^{\circ}$. Sendo que, no GS, a média das mensurações angulares da cifose torácica, por meio do método Flexicurva, foi de $48,60^{\circ}$ e desvio-padrão de $\pm 10,46$. E no GAF, a média das mensurações angulares da cifose torácica, por meio do método Flexicurva, foi de 46,18 e desvio padrão de \pm 9,91 (Tabela 1).

Apesar da média de cifose torácica do GS $\left(48,60^{\circ} \pm 10,46\right)$ ter sido superior a do GAF $\left(46,18^{\circ}\right.$ $\pm 9,91)$, não houve diferença significativa entre elas [ $\mathrm{t}(38)=0,75 ; \mathrm{p}=0,45]$, indicando que idosos ativos e sedentários possuem características similares quanto à cifose torácica.
Com base no grau da curvatura da cifose torácica, identificou-se que a média total das mensurações angulares das cifoses torácicas foi menor que o nível considerado fisiológico (curvatura de até $\left.56^{\circ}\right)^{7,8}$. Desta forma, verificou-se que os grupos possuem características similares quando se compara a cifose torácica de até $56^{\circ}$ e acima de $56^{\circ}$. (Tabela 2).

Tabela 1. Distribuição da amostra por grupo, média de idade e desvio padrão dos valores obtidos em graus com o método Flexicurva.

\begin{tabular}{lcccc}
\hline & $\mathrm{n}$ & $\begin{array}{c}\text { Média de } \\
\text { Idades } \\
\text { (anos) }\end{array}$ & $\begin{array}{c}\text { Curvatura Ci- } \\
\text { fose Torácica }\end{array}$ & $\begin{array}{c}\text { Desvio } \\
\text { Padrão da } \\
\text { Curvatura Ci- } \\
\text { fose Torácica }\end{array}$ \\
\hline GS & 20 & 64,4 & $48,60^{\circ}$ & $\pm 10,46$ \\
GAF & 20 & 66,75 & $46,18^{\circ}$ & $\pm 9,91$ \\
$\begin{array}{l}\text { Média } \\
\text { Total }\end{array}$ & 20 & 65,5 & $47,39^{\circ}$ & $\pm 10,13$ \\
\hline
\end{tabular}

GS = sedentários; GAF = praticantes de atividade física.

Tabela 2: Percentual da cifose torácica entre os grupos de sedentário e grupo de praticante de atividade física.

\begin{tabular}{lll}
\hline $\begin{array}{l}\text { Curvatura } \\
\text { Cifose Torácica }\end{array}$ & GS & GAF \\
\hline Até $56^{\circ}$ & $75 \%$ & $75 \%$ \\
Acima de $56^{\circ}$ & $15 \%$ & $15 \%$ \\
\hline
\end{tabular}

$\mathrm{GS}=$ sedentários; GAF = praticantes de atividade física.

\section{DISCUSSÃO}

Foi escolhido o Método Flexicurva no presente estudo, pois é um método que não expõe o paciente à radiação, não é invasivo, de fácil aplicabilidade e serve como forma de diagnóstico e indicador de evolução de tratamento em idosos. $\mathrm{O}$ estudo de Souza ${ }^{20}$ observou uma amostra de 47 indivíduos, que o Método Flexicurva comparado com o Método de Cobb apresentam uma boa sensibilidade $(52,63 \%)$ e boa especificidade $(82,14 \%)$ e uma correlação positiva e estaticamente significativa com um $\mathrm{r}=0,866$ ( $\mathrm{p}=0,0001)$.

No presente estudo, observou-se que a cifose torácica das idosas $\left(47,50^{\circ} \pm 9,56\right)$ é mais acentuada se comparada com a dos idosos $\left(46,38^{\circ} \pm 8,71\right)$. Fon et al. ${ }^{8}$ confirmam que a cifose torácica se acentua com o passar dos anos, principalmente, em mulheres. Segundo Souza ${ }^{20}$ muitas alterações da curvatura cifótica podem estar associadas a fatores ambientais e ocupacionais. No presente estudo, apesar da média de cifose torácica do GS $\left(48,60^{\circ} \pm 10,46\right)$ ter sido superior a do GAF $\left(46,18^{\circ} \pm 9,91\right)$, não houve diferença significativa entre elas $[\mathrm{t}(38)=0,75 ; \mathrm{p}=0,45]$, 
indicando que idosos ativos e sedentários possuem características similares quanto à cifose torácica. Hinma $^{21}$ salienta em seu estudo da comparação da cifose torácica em mulheres jovens e idosas que a fraqueza muscular pode afetar o alinhamento postural, afirmando que a cifose torácica é maior em mulheres idosas. No estudo de Ettinger et al.22 , ao mensurar o grau de cifose torácica e sua correlação com a densidade mineral óssea, verificou-se que esta é uma das responsáveis pelo aumento do grau de cifose, ou seja, quanto menor a densidade óssea maior o grau desta cifose torácica.

$\mathrm{Na}$ caracterização da amostra apresentada nessa pesquisa, observou-se que, dos 40 idosos entrevistados, houve uma predominância do sexo feminino (90\%), na faixa etária de 60 a 69 anos de idade. A predominância de mulheres no estudo foi devido a UnATI apresentar em torno de 85\% desta população. Desta forma, verificou-se (Tabela 2) que a média de idade foi maior no grupo de pacientes praticantes de atividades físicas (66,75 anos).

No presente estudo, obteve-se uma média total das mensurações angulares da cifose torácica dos dois grupos, por meio do Método Flexicurva, de 47,39 ${ }^{\circ}$, assim, os valores apresentados encontram-se dentro dos parâmetros estipulados como normal de $20^{\circ}$ a $56^{\circ}$. Fon et al. ${ }^{8}$, em seu estudo, analisaram uma amostra de 316 pessoas, e obtiveram valores médios de $44,86^{\circ}$ para mulheres e $34,67^{\circ}$ para homens com idade entre 60 e 69 anos.

Após a análise dos dados, observou-se, também (Tabela 1), que: no GS, a média das mensurações angulares da cifose torácica por meio do Método Flexicurva foi de $48,60^{\circ}$, enquanto no GAF, a média foi de $46,18^{\circ}$. $\mathrm{Kura}^{23}$, em seu estudo, com uma amostra de 315 idosos, apresentou maior grau da curvatura da cifose torácica no grupo de idosos sedentários. Segundo Ettinger et al. ${ }^{22}$ a inatividade física e funcional associada ao envelhecimento, acarreta em alterações na curvatura da coluna torácica, desta forma, este efeito pode ser minimizado através da atividade física regular que contribui para evitar as incapacidades associadas ao envelhecimento. Seu enfoque principal deve ser na promoção de saúde, mas em indivíduos com patologias já instaladas a prática de exercícios orientados pode ser muito importante para controlar a doença, evitando sua progressão.

Quando comparado o percentual da cifose torácica entre os grupos de sedentário e grupo de praticante de atividade física não houve diferença significativa entre os praticantes de atividades físicas e os sedentários desse estudo, mas a atividade física pode estar influenciando de outras formas na cifose torácica (Tabela 2). Tini ${ }^{24}$ ressalta que a manutenção de uma atividade física regular, ao longo da vida, pode colaborar para a manutenção ideal do peso, para menores índices de percentual de gordura e IMC, além de contribuir para a minimização das perdas de massa muscular. Ettinger et al. ${ }^{22}$ confirmam que o declínio físico e funcional associado ao envelhecimento, mesmo em pessoas com idade avançada, pode ser atenuado através do exercício físico.

\section{CONCLUSÃO}

O resultado obtido mostra que há uma tendência dos idosos praticantes de atividade física em apresentar menor grau de cifose torácica, quando comparados aos sedentários, porém, estatisticamente, não foi significativa a diferença nesse estudo.

Os idosos praticantes de atividade física apresentam fatores que favorecem para uma curvatura fisiológica, desta forma, a inclusão do idoso em um programa de exercícios físicos regular, possibilita a prevenção efetiva de perdas funcionais associadas ao envelhecimento.

Os resultados deste estudo são importantes para a saúde pública, à medida que podem ser utilizados para gerenciar programas de incentivo à prática de atividades físicas, na perspectiva de prevenção das internações hospitalares e, consequentemente, na redução de gastos para o sistema de saúde.

\section{REFERÊNCIAS BIBLIOGRÁFICAS}

1. Instituto Brasileiro de Geografia e Estatística (IBGE). Contagem da população 2007. Rio de Janeiro 2007.

2. Tribess S, Virtuoso JS. Prescrição de exercícios físicos para idosos. Rev Saúde. Com 2005;1(2):163-72.

3. Figueiredo KM, Lima KC. Instrumentos de avaliação do equilibrio corporal em idosos. Rev Bras Cineantropom Desempenho Hum 2007;9(4):408-13.

4. Geraldes A, Cavalcante AP. Correlação entre a flexibilidade multiarticular e o desempenho funcional de idosas fisicamente ativas em tarefas motoras selecionadas. Rev Bras Cineantropom Desempenho Hum 2007;9(3):238-43.

5. Cutler W, Friedmann E, Genovese-stone E. Prevalence of kyphosis in a healthy sample of pre and post menopausal women. Am J Phys Med Rehabil 1993;72:219-25.

6. Colledge NR, Cantley P, Peaston I, Brash H, Lewis S, Wilson, Culham E. Thoracic Kyphosis, rib mobility and lung volumes in normal women and women with osteoporosis. Spine 1994; 19(11):1250-55.

7. Bradford DS, Lonstein JE, Moe JH, Ogilvie JW, Winter RB. Escolioses e outras deformidades da coluna: "O livro de Moe". $2^{a}$ ed. São Paulo: Santos; 1994. 
8. Fon G, Pitt M, Thies A. Thoracic kyphosis: range in normal subjects. AJR 1980;134: 979-83.

9. Poolman R, Been H, Ubags L. Clinical outcome and radiographic results after operative treatment of Scheuermann's disease. Eur Spine J 2002;11:561-69.

10. Hallal PC, Victora CG, Wells JCK, Lima RC. Physical inactivity: prevalence and associated variables in Brazilian adults. Med Sci Sports Exerc 2003;35(11):1894-900.

11. Teixeira FA, Carvalho GA. Confiabilidade e validade das medidas da cifose torácica através do método flexicurva. Rev Bras Fisioter 2007;11(3):199-204.

12. Shumway-cook A, Brauer S, Woollacott M. Predicting the probability for falls in community-dwelling older adults using the timed Up \& Go test. Physical Therapy, 2000;80(9):896-903.

13. Carvalho AM, Coutinho ESF. Demência como fator de risco para fraturas graves em idosos. Rev Saúde Pública 2002;36(4)448-54.

14. Perracini MR, Ramos LR. Fatores associados a quedas em uma coorte de idosos residentes na comunidade. Rev Saúde Publica 2002;36(6):709-16.

15. Cohen BR, Wolf SL. Environmental and Behavioral circumstances associated with falls at home among helthy elderly individuals. Archives Physical Med Rehabilitation, Philadlphia 1997;78:179-86.

16. Monteiro WD, Amorim RS, Farjalla R, Farinatti TV. Força muscular e características morfológicas de mulheres idosas praticantes de um programa de atividades físicas. Rev Bras Ativ fís saúde 1999;4(1):320-31.

17. Pereira MG. Epidemiologia teoria e prática. $8^{\mathrm{a}}$ ed. Rio de Janeiro: Koogan; 2005.

18. Marques AP, Peccin MS. Pesquisa em fisioterapia: a pratica baseada em evidências e modelos de estudos. Rev Fisioterapia Pesquisa 2005;11(1):43-8.

19. World Health Organization. International Physical Activity Questionnaires. IPAQ Scoring Protocol. Geneva. 2001. http://www.ipaq.ki.se/IPAQ.asp?mnu_ sel=EE\&pg_sel=CCB.12 n. [2008 Aug 08].
20. Souza DP. Avaliação angular da cifose torácica em idosos: estudo correlacional entre o método flexicurva e o método de COBB. [Dissertação de Mestrado-Programa de Pós-graduação em Gerontologia]. Brasília (DF): Universidade Católica de Brasília; 2006.

21. Hinman M. Comparatios of thoracic kyphosis andpostural stiffnes in younger and older women. Spine Jing J 2004:4:413-17.

22. Ettinger B, Black DM, Palermo L, Nevitt MC, Melnikoff S, Cummings SR. Kyphosis in older women and its relation to back pain, disability and osteopenia: the study of osteoporotic fractures. Osteoporps Int 1994:4:55-60.

23. Kura GG. Nível de atividade física, IMC e índices de forças musculares estática entre idosas praticantes de hidroginástica e ginástica. Rev Bras Ciênc Envelhecimento Hum 2003;1(2):30-40.

24. Tini VGNS. Correlação entre o grau da cifose e a flexibilidade em idosos praticantes de atividade física. [Dissertação de Mestrado-Programa de Pós-graduação em Gerontologia]. Brasília (DF): Universidade Católica de Brasília; 2007.

\section{Endereço para correspondência}

Fabrício Marinho Bandeira

QNL 30 Via Ln 30 Casa 32 - Taguatinga Norte

CEP: 72.162-330 - Brasília, DF. Brasil.

E-mail: fabriciomarinho3@hotmail.com 